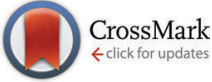

Cite this: Phys. Chem. Chem. Phys., 2015, 17, 14750

Received 10th February 2015, Accepted 29th April 2015

DOI: $10.1039 / c 5 c p 00851 d$

www.rsc.org/pccp

\section{The effect of anaesthetics on the properties of a lipid membrane in the biologically relevant phase: a computer simulation study $\dagger$}

\author{
Balázs Fábián, ${ }^{a b}$ Mária Darvas, ${ }^{c}$ Sylvain Picaud, ${ }^{d}$ Marcello Sega ${ }^{e}$ and \\ Pál Jedlovszky*afg
}

\begin{abstract}
Molecular dynamics simulations of the fully hydrated neat dipalmitoylphosphatidylcholine (DPPC) membrane as well as DPPC membranes containing four different general anaesthetic molecules, namely chloroform, halothane, diethyl ether and enflurane, have been simulated at two different pressures, i.e., at 1 bar and 1000 bar, at the temperature of $310 \mathrm{~K}$. At this temperature the model used in this study is known to be in the biologically most relevant liquid crystalline $\left(L_{\alpha}\right)$ phase. To find out which properties of the membrane might possibly be related to the molecular mechanism of anaesthesia, we have been looking for properties that change in the same way in the presence of any general anaesthetic molecule, and change in the opposite way by the increase of pressure. This way, we have ruled out the density distribution of various groups along the membrane normal axis, orientation of the lipid heads and tails, self-association of the anaesthetics, as well as the local order of the lipid tails as possible molecular reasons of anaesthesia. On the other hand, we have found that the molecular surface area, and hence also the molecular volume of the membrane, is increased by the presence of any anaesthetic molecule, and decreased by the pressure, in accordance with the more than half a century old critical volume hypothesis. We have also found that anaesthetic molecules prefer two different positions along the membrane normal axis, namely the middle of the membrane and the outer edge of the hydrocarbon region, close to the polar headgroups. The increase of pressure is found to decrease the former, and increase the latter preference, and hence it might also be related to the pressure reversal of anaesthesia.
\end{abstract}

\section{Introduction}

Ever since the first application of anaesthesia in surgery it is known that its molecular mechanism is somehow related to the cell membrane. The assumption that general anaesthetics are dissolved in the cell membrane was first confirmed experimentally

\footnotetext{
${ }^{a}$ Laboratory of Interfaces and Nanosize Systems, Institute of Chemistry, Eötvös Loránd University, Pázmány P. Stny 1/A, H-1117 Budapest, Hungary. E-mail:pali@chem.elte.hu

${ }^{b}$ Department of Inorganic and Analytical Chemistry, Budapest University of Technology and Economics, Szt. Gellért tér 4, H-1111 Budapest, Hungary

${ }^{c}$ SISSA, Sector of Molecular and Statistical Biophysics, 265 via Bonomea, 34136 Trieste, Italy

${ }^{d}$ Institut UTINAM (CNRS UMR 6213), Université de Franche-Comté, 16 route de Gray, F-25030 Besançon, France

${ }^{e}$ Institut für Computergestützte Biologische Chemie, University of Vienna, Währinger Strasse 17, A-1090 Vienna, Austria

${ }^{f}$ MTA-BME Research Group of Technical Analytical Chemistry, Szt. Gellért tér 4, H-1111 Budapest, Hungary

${ }^{g}$ EKF Department of Chemistry, Leányka u. 6, H-3300 Eger, Hungary

$\dagger$ Electronic supplementary information (ESI) available. See DOI: 10.1039/ c5cp00851d
}

by Meyer ${ }^{1}$ and Overton, ${ }^{2}$ who showed that there is a linear dependence of anaesthetic efficiency and the oil/water partition coefficient of the general anaesthetic molecules. However, in spite of the ongoing scientific investigation of the problem for more than a hundred years, the molecular mechanism of general anaesthesia is still far from being understood. The difficulty in explaining the mechanism of the action of general anaesthetics originates from the fact that the chemical structure, shape, size, and even polarity of the general anaesthetics scatter in a rather broad range, and any possible explanation has to account for the effect of all general anaesthetics. The problem is further complicated by the experimentally well known fact that the effect of general anaesthetics is reverted by the increase of pressure. ${ }^{3-6}$ Therefore, any possible explanation of the mechanism of general anaesthesia has to account also for its pressure reversal.

Possible explanations of this phenomenon can be divided into two main groups. Some of these explanations assume that anaesthesia is related to certain changes in the structure and/or dynamics of the cell membrane itself, caused by the presence of general anaesthetic molecules. ${ }^{7-13}$ Other explanations assume 
that general anaesthetics alter the function of several channel forming proteins that are embedded in the membrane. ${ }^{14-18}$ However, considering the fact that protein-substrate interactions usually correspond to strong specific binding, and also the large chemical variety of general anaesthetics, the role of any direct protein-anaesthetics interaction seems to be very unlikely in this respect. Therefore, even if anaesthetics alter the structure, and hence the function of certain membrane proteins, it is expected to be done indirectly, by altering primarily the membrane properties.

Concerning the changes induced by the presence of general anaesthetics in the membrane structure and dynamics, several suggestions have been made in the past six decades. Thus, among others, anaesthetic molecules were found to increase the membrane thickness by ordering the lipid tails, ${ }^{13,19,20}$ and increase the fluidity of the membrane. ${ }^{6,21}$ The critical volume hypothesis of Mullins assumes that by increasing the orientational order of the lipid tails, and hence making these tails straighter, general anaesthetics increase the thickness of the membrane, and anaesthesia occurs when the molar volume of the membrane exceeds a critical value. ${ }^{7}$ This hypothesis can easily account for pressure reversal, since the increase of the pressure leads to the decrease of the molar volume. A more recent explanation of Cantor conjectures that the changes in the lateral pressure profile induced by the presence of the anaesthetic molecules alter the equilibrium conformation of some channel forming proteins, and this change is responsible for anaesthesia. ${ }^{22}$ Since this explanation is based on changes in the pressure profile, it can, in principle, also account for the phenomenon of pressure reversal.

Experimental findings are, however, rather controversial in all these respects. Thus, in spite of the above claims concerning the effect of general anaesthetics on the membrane properties, several experimental studies have been published in which the membrane thickness turned out to be insensitive to the presence or absence of a general anaesthetic, ${ }^{23,24}$ and some general anaesthetics were found not to alter or even decrease $\mathrm{e}^{23,25}$ the order of the lipid tails.

Although experimental studies can be well complemented by computer simulations, and the mechanism of general anaesthesia has been in the focus of computer simulation investigations in the past two decades, the results of these studies are not less controversial than those of the experiments. Thus, several authors reported both a slight increase $\mathrm{e}^{26-28}$ and decrease $\mathrm{e}^{29,30}$ of the order of the lipid tails or parts of them in the presence of general anaesthetics, although in some cases this effect was too small to be considered as statistically significant. ${ }^{26,27}$ Apart from a slight ordering of the lipid tails, Oh and Klein did not find any effect of halothane molecules on the properties of a dimyristoylphosphatidylcholine (DMPC) bilayer, in accordance with an earlier experimental claim of Franks and Lieb, ${ }^{17,23}$ even if the halothane concentration in their simulation well exceeded that of surgical conditions. ${ }^{28}$ Huang et al. found some indications that anaesthetic molecules induce structural changes leading to an increased membrane fluidity, ${ }^{26}$ again in accordance with earlier experimental claims. ${ }^{6,21}$ Chau et al. proposed that anaesthetic molecules might block the binding site of some membrane proteins, but the increase of the pressure induces self-aggregation of these molecules. They hypothesized that pressure reversal is caused by the fact that such self-aggregates are too large to block these binding sites. ${ }^{27,31}$ No sign of such pressure induced self-association of anaesthetic molecules was, on the other hand, found in a recent study of ours in a membrane being below the gel-liquid crystalline phase transition. ${ }^{32}$ Griepernau and Böckmann calculated the lateral pressure profile in neat and anaesthetic containing membranes at low and high pressures, ${ }^{33}$ and found results that are compatible with the earlier claim of Cantor. ${ }^{22}$

Another important issue concerns the preferred location of the general anaesthetic molecules along the membrane normal axis. By calculating the free energy profile of chloroform across a neat DMPC membrane ${ }^{34}$ as well as in DMPC-cholesterol mixed membranes of various compositions, ${ }^{35}$ Jedlovszky and Mezei showed that chloroform prefers to stay right in the middle of the membrane. Similar conclusions were drawn by Chau et al. concerning the preferred location of halothane. ${ }^{27,31}$ On the other hand, Klein et al. found by simulating halothane containing dipalmitoylphosphatidylcholine (DPPC) membranes that the preferred location of halothane is in the hydrocarbon phase, but close to the region of the polar headgroups rather than in the middle of the membrane..$^{30,36,37}$ Similar conclusions were drawn earlier by Huang et al. concerning trichloro ethylene as the general anaesthetic, ${ }^{26}$ and also very recently by Martin et al. for two local anaesthetic molecules. ${ }^{38}$ These claims were supported by subsequent experimental results of Hauet et al., who studied enflurane in the DPPC membrane. ${ }^{39}$ Porasso et al. demonstrated that the preferred location of the anaesthetic molecules depends also on the lipid molecules constituting the membrane. ${ }^{40}$ Recently we proposed that, at least in the biologically less relevant gel phase, general anaesthetic molecules have a dual preference, and hence locations close to the polar headgroup region and in the middle of the membrane are both preferred. ${ }^{32}$

Some of the above contradictions can be attributed to the insufficient sampling (i.e., too short simulation time, too small system size, and too few anaesthetic molecules) in some of the earlier simulations. Thus, the total simulation time, including equilibration was below $1 \mathrm{~ns},{ }^{26}$ around $1 \mathrm{~ns}^{27,29,31}$ and around $2 \mathrm{~ns}^{30,36}$ in several studies, in spite of the more than ten-year-old finding of Anézo et al. that reliable simulations of phospholipid membranes require at least $10-20$ ns equilibration. ${ }^{41}$ Further, in some early studies the number of the phospholipid and anaesthetic molecules did not exceed 36 and 3, respectively. ${ }^{26,29}$

However, another important source of the seemingly controversial results might well be that different anaesthetic molecules behave differently inside the membrane, and hence modify the membrane properties in different ways. On the other hand, the phenomenon of general anaesthesia can only be explained by changes induced in the membrane structure by any possible general anaesthetic molecule. Further, because of the pressure reversal this change should be such that the increase of the pressure must have an opposite effect on the membrane properties. Considering this fact we performed recently a systematic study of the effect of both the pressure and the presence of four 
general anaesthetic molecules of different size, shape and polarity on the properties of the DPPC membrane in the gel phase, i.e., slightly below the gel-liquid crystalline phase transition of the potential model used. ${ }^{32}$ We found that the average area per headgroup and, consequently, the molar volume of the membrane is increased by all the four anaesthetic molecules considered, and decreased by the increase in the pressure, whereas no such systematic influence of the anaesthetics and pressure was found on other membrane properties. ${ }^{32}$

In the present paper we extend this study to the biologically more relevant liquid crystalline $\left(\mathrm{L}_{\alpha}\right)$ phase. Thus, we report molecular dynamics simulations of the fully hydrated neat DPPC bilayer as well as DPPC bilayers containing four different general anaesthetic molecules, namely chloroform (CF), halothane (HAL), diethyl ether (DE), and enflurane (ENF), both at atmospheric pressure and at 1000 bar. All these simulations are performed at body temperature, i.e., $310 \mathrm{~K}$, where the model used is well in the liquid crystalline $\left(\mathrm{L}_{\alpha}\right)$ phase. The schematic structure of the molecules used in the simulations is shown in Fig. 1. The general anaesthetic molecules have been chosen in such a way that their shape ranges from almost spherical (CF) to chain-like (DE), their size ranges from 4 (CF) to 11 (ENF) heavy (i.e., non-hydrogen) atoms, their mass ranges from 74 (DE) to almost 200 (HAL) atomic units, their molecular volume ranges from about 130 (CF) to $200 \AA^{3}$ (ENF), and their molecular dipole moment ranges from about 1 (CF) to $2 \mathrm{D}$ (HAL). To magnify any possible effect of both the anaesthetic molecules and pressure on the membrane properties we follow the approach of $\mathrm{Oh}$ and Klein, ${ }^{28}$ and use considerably higher anaesthetic concentrations and pressure than what is needed to perform anaesthesia and its pressure reversal, respectively. This way, their effects on the membrane properties are exaggerated, making systematic changes better distinguishable from statistical noise. To avoid artefacts due to insufficient equilibration or sampling we performed about an order of magnitude longer simulations than in the majority of the studies reported in this field. We investigate the effect of the presence of anaesthetic molecules as well as that of high pressure on several different properties of the membrane, including its thickness and average area per headgroup, distribution of various groups along the

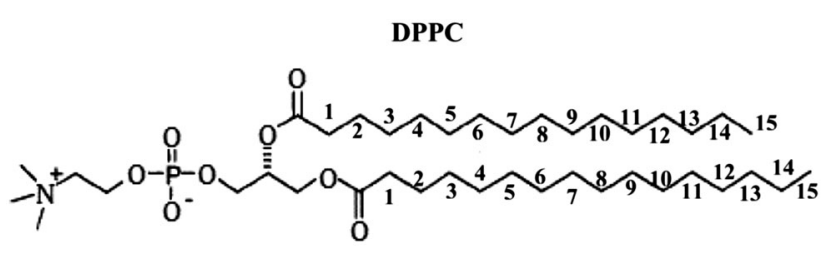<smiles>ClC(Cl)Cl</smiles>

CF

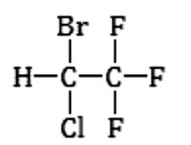

HAL

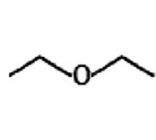

DE

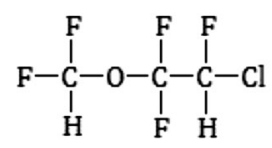

ENF
Fig. 1 Schematic structure of the lipid and anaesthetic molecules used in the simulations. The numbering scheme of the lipid tail $\mathrm{C}$ atoms used throughout this paper is also indicated. membrane normal axis, orientational preferences of the lipid tails and headgroups, and the local order of the lipid tails. In addition, the distribution of the anaesthetic molecules along the membrane normal axis as well as their possible pressure induced self-association is also investigated.

Our approach is rather simple. We are looking for membrane properties that are (i) affected by all the four anaesthetic molecules considered in the same way and (ii) affected by the increase of the pressure in the opposite way, as only such membrane properties can be behind any possible explanation of the molecular mechanism of anaesthesia and its pressure reversal. On the other hand, all other properties of the membrane (i.e., that are altered by different general anaesthetic molecules in different ways, or altered by the increase of the pressure in the same way as by the presence of the anaesthetics) can be excluded from the explanation of the mechanism of anaesthesia.

The paper is organised as follows. In Section 2 details of the calculations performed are given. The obtained results are presented and discussed in detail in Section 3. Finally, in Section 4 the main conclusions of this study are summarised.

\section{Computational details}

\subsection{Molecular dynamics simulations}

Molecular dynamics simulations of the fully hydrated neat DPPC bilayer as well as DPPC bilayers containing chloroform, halothane, diethyl ether and enflurane have been performed on the isothermal-isobaric $(N, p, T)$ ensemble at the temperature of $310 \mathrm{~K}$. All the five systems have been simulated at two different pressures, namely at 1 bar and at 1000 bar. The systems simulated consisted of 256 DPPC and 8132 water molecules (the water/DPPC ratio being well above the value of 29.1, required for full hydration ${ }^{42}$ ), and the respective systems contained $112 \mathrm{CF}, 72 \mathrm{HAL}, 192 \mathrm{DE}$, and 96 ENF molecules, making the anaesthetic mass density roughly equal in the different systems simulated. In order to exaggerate the effects to be studied, the used anaesthetic concentrations well exceed those employed under surgical conditions, and the applied pressure of 1000 bar also exceeds the few hundred bars needed for pressure reversal. To check the validity of this approach, first suggested by $\mathrm{Oh}$ and Klein, ${ }^{28}$ we have repeated the simulations of the membranes containing CF and HAL at the pressure of 100 bar, and also the 1 and 1000 bar simulations of the halothane containing membrane with half of the original halothane concentration (i.e., using 36 instead of $72 \mathrm{HAL}$ molecules). The obtained results have always been consistent with the above approach of Oh and Klein, as the observed effects have indeed been magnified but not altered by the exaggeration of the anaesthetic concentration and pressure.

DPPC molecules have been modelled by the GROMOS87based force field, developed specifically for lipids by Berger et al. ${ }^{43}$ At the simulation temperature of $310 \mathrm{~K}$ the DPPC bilayer described by this potential model is known to be above the gelliquid crystalline transition, in the biologically more relevant liquid crystalline $\left(\mathrm{L}_{\alpha}\right)$ phase (the transition temperature of this 
model being $305 \mathrm{~K}) .{ }^{44}$ Water and halothane molecules have been described by the rigid, three site SPC potential ${ }^{45}$ and by the potential model proposed by Scharf and Laasonen, ${ }^{46}$ respectively, whereas for the description of the other anaesthetic molecules the

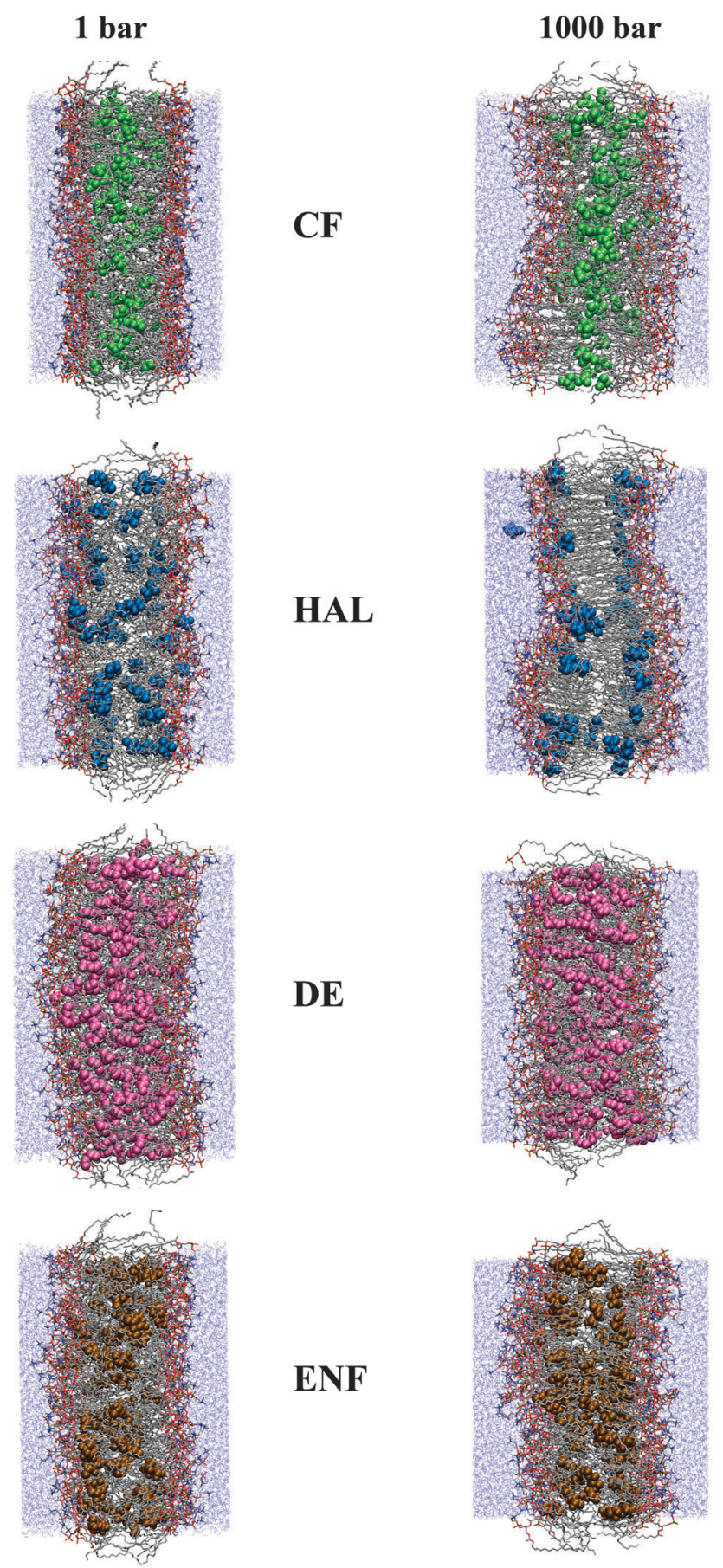

Fig. 2 Equilibrium snapshots of the DPPC membranes containing CF (top row), HAL (second row), DE (third row), and ENF (bottom row), as taken out from the simulations performed at 1 bar (first column) and 1000 bar (second column). The $\mathrm{O}, \mathrm{N}, \mathrm{P}$ and $\mathrm{C}$ atoms of the lipid molecules are represented by red, dark blue, yellow, and grey colours, respectively, whereas $\mathrm{H}$ atoms are omitted from the snapshots. Water molecules are shown by thin, dark cyan sticks. For better visibility, CF, HAL, DE, and ENF molecules are shown enlarged, by green, blue, pink, and brown colours, respectively.
GROMOS96 force field ${ }^{47-50}$ has been used. All the used potential models are pairwise additive, hence, the intermolecular part of the total potential energy of the system is simply the sum of the interaction energy of all molecule pairs, and the interaction energy of a molecule pair is calculated as the sum of the Lennard-Jones and charge-charge Coulomb contributions of all pairs of their interaction sites. $\mathrm{CH}, \mathrm{CH}_{2}$ and $\mathrm{CH}_{3}$ groups are treated by these models as united atoms. All bond lengths have been kept fixed in the simulations by means of the LINCS algorithm, ${ }^{51}$ with the exception of the water molecules, which have been kept completely rigid using the SETTLE ${ }^{52}$ method. All interactions have been truncated to zero beyond the group-based cut-off distance of $9.0 \AA$; the long range part of the electrostatic interactions has been accounted for using the Particle Mesh Ewald summation method ${ }^{53}$ in its smooth (sPME) variant.

The simulations have been performed using the GROMACS 4.5.5. program package. ${ }^{54}$ Starting configurations have been taken from our previous simulations. ${ }^{32}$ The equations-of-motion have been integrated in time steps of 2 fs. The temperature and pressure of the systems have been controlled by means of the Nosé-Hoover ${ }^{55,56}$ and Parrinello-Rahman ${ }^{57}$ algorithms, respectively, with the characteristic time of $0.5 \mathrm{ps}$ in both cases. Pressure has been controlled using a semi-isotropic coupling, i.e., separate couplings in the plane of the membrane and along the membrane normal axis. The systems have been equilibrated for $20 \mathrm{~ns}$ each, in accordance with the suggestion of Anézo et l. $^{41}$ In the subsequent 20 ns long production stage, 2000 equilibrium configurations per system, separated from each other by 10 ps long trajectories each, have been dumped for the analyses. To demonstrate that the systems have been properly equilibrated, the evolution of the total energy of the systems simulated at 1 bar during the entire course of the simulations, and that of the volume and cross section area of the basic box of these systems during the production stage of the simulations are shown in Fig. S1 of the ESI. $\dagger$ An equilibrium snapshot of the systems containing anaesthetic molecules both at 1 bar and at 1000 bar are shown in Fig. 2, as taken out from the simulations.

\section{Results and discussion}

\subsection{Density profiles}

3.1.1. Lipid density profiles. The mass density profile of the five simulated membranes are shown in Fig. 3 as obtained both at 1 bar and 1000 bar, whereas Fig. 4 compares these profiles with the number density profiles of selected lipid atoms. The electron density profile obtained for the pure DPPC membrane at 1 bar compares reasonably well with available experimental data ${ }^{42}$ (see the inset of Fig. 3). As is seen, the presence of the anaesthetic molecules has no clear influence on the mass density profile. Thus, at 1 bar, the density in the middle of the membrane is increased by the presence of HAL and ENF; however, it turned out to be insensitive to the presence of $\mathrm{CF}$, and even decreased slightly in the presence of DE. Similarly, HAL and ENF turn out to increase, whilst CF and DE to decrease the membrane density at a distance of about 

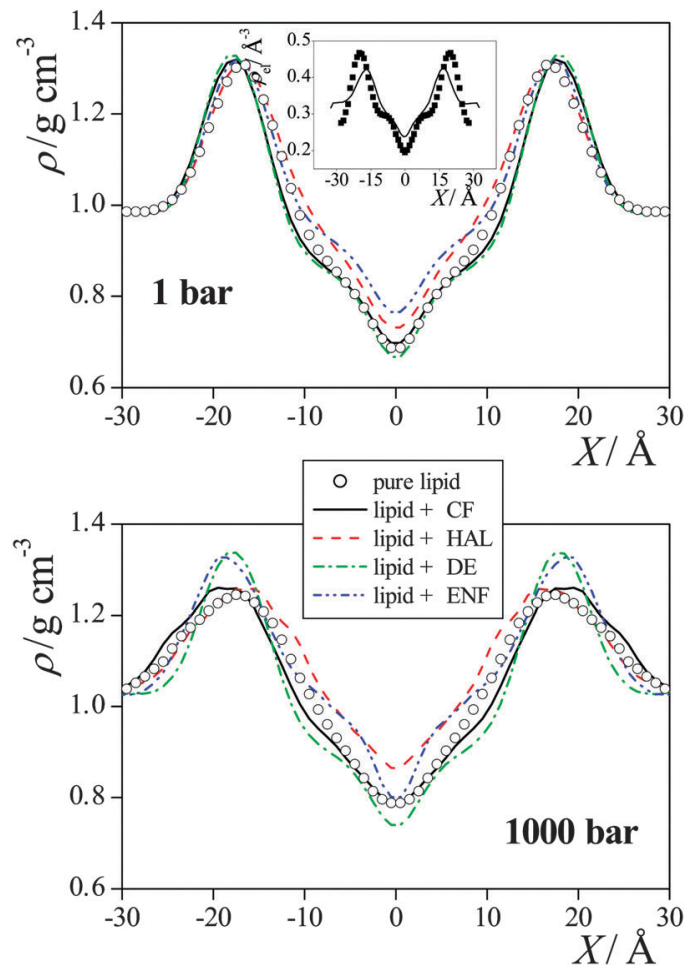

Fig. 3 Mass density profile of the DPPC membranes simulated without anaesthetics (open circles), with CF (black solid lines), with HAL (red dashed lines), with DE (green dash-dotted lines), and with ENF (blue dash-dot-dotted lines) at 1 bar (top panel) and at 1000 bar (bottom panel). The inset shows a comparison of the simulated electron density profile of the neat DPPC membrane at 1 bar (solid line) with the experimental data of Nagle et al. ${ }^{42}$ (full squares). The profiles are averaged over the two leaflets of the membrane. Error bars are below $0.01 \mathrm{~g} \mathrm{~cm}^{-3}$.

$10 \AA$ from the membrane center, i.e., at $X \sim \pm 10 \AA$, at the outer part of the hydrocarbon region, in the vicinity of the polar headgroups (see Fig. 4). Further, the different anaesthetic molecules have the same, although considerably more marked effect on the membrane density profile at 1000 bar than at 1 bar. The position and density of the polar headgroup region is found to be rather insensitive to the presence or absence of anaesthetic molecules at 1 bar, whereas at 1000 bar the presence of DE and ENF have led to a noticeable increase in the headgroup density. The position of the density peak in the headgroup region, located at $X= \pm 17.0 \AA$ in the neat DPPC membrane with both pressures considered, has moved slightly outward in the presence of anaesthetics.

In general, the increase of the pressure has led to a more uniform mass distribution along the membrane normal axis, as it has decreased the density around its maximum in the headgroup region, and increased it in the low density region of the hydrocarbon tails (see Fig. 4). However, there are exceptions also in this respect, as the headgroup density has not changed by the pressure increase in the presence of DE and ENF. No systematic effect of the presence or absence of anaesthetics as well as that of the pressure is seen on the density profiles of the various lipid atoms either.

3.1.2. Anaesthetic density profiles. The mass density profiles of the anaesthetic molecules are also shown in Fig. 4 both
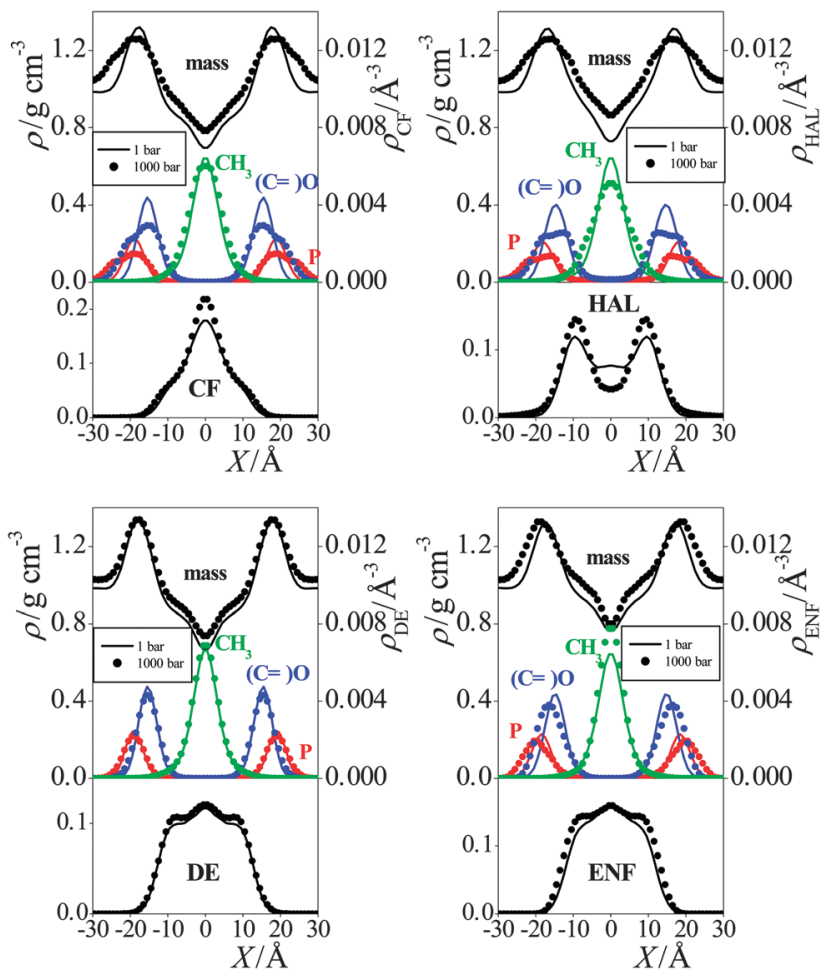

Fig. 4 Mass density profiles of the simulated membranes (top panels), number density profiles of selected lipid atoms: headgroup P (red), oxo $O$ of the ester groups (blue) and chain terminal C (green) (middle panels), and mass density profiles of the anaesthetic molecules (bottom panels) in the membranes containing CF (top left graph), HAL (top right graph), DE (bottom left graph), and ENF (bottom right graph) at the pressures of 1 bar (solid lines) and 1000 bar (full circles). The profiles are averaged over the two leaflets of the membrane. Error bars are below $0.01 \mathrm{~g} \mathrm{~cm}^{-3}$ and $0.0001 \AA^{-1}$

at 1 bar and 1000 bar. The obtained profiles indicate two preferred positions of the anaesthetic molecules along the membrane normal axis in every case. Thus, at 1 bar the density profiles of $\mathrm{CF}, \mathrm{ENF}$ and $\mathrm{DE}$ have their peak at $X=0 \AA$, i.e., right at the middle of the membrane, but exhibit a clear shoulder around the $X$ values of $\pm 10 \AA$, this shoulder being the strongest and weakest when $\mathrm{DE}$ and $\mathrm{CF}$, respectively, is used as an anaesthetic. As is seen from Fig. 4, the location of this shoulder is close to the position of the double bonded ester $\mathrm{O}$ atoms of the lipid tails, which marks the boundary between the regions of the apolar tails and polar headgroups. Moreover, the density profile of HAL has its two main peaks at $X= \pm 10 \AA$, and only a small, secondary peak occurs in the middle of the membrane.

The preference of the anaesthetic molecules for being in the middle of the membrane can be simply explained by steric effects, as this is clearly the lowest density part of the membrane (see Fig. 3). On the other hand, if the anaesthetic molecule is located in the apolar part of the membrane but close enough to the headgroup region, its small dipole moment might favourably interact with the nearby headgroups. This effect is particularly strong in the case of the HAL molecule, the exceptionally strong preference of which for this region can be explained by the fact that its $\mathrm{CH}$ group, linked to two atoms of 
high electronegativity (see Fig. 1), can form a weak, C-H. . O type hydrogen bond with both the oxo (i.e., $=\mathrm{O}$ ) and ether (i.e., -O-) oxygen atoms of the ester groups linking the lipid tails to the glycerol backbone. ${ }^{32}$

The most evident change induced by the pressure increase on the anaesthetic density profiles is an overall increase of the density, while it leaves the above dual preference of the anaesthetic molecules basically unchanged, although the shoulders at $\pm 10 \AA$ of the DE and ENF profiles turn into separate peaks at 1000 bar. The only exception in this respect is the profile of HAL, on which the small maximum located at $X=0 \AA$ at 1 bar turns into a minimum at 1000 bar.

To further investigate this point, we have fitted the sum of three Gaussian functions to the obtained anaesthetic density profiles at both pressures considered. Since the density profiles calculated in the simulations are always symmetrised over the two leaflets of the membrane, the middle Gaussian of this fit is always placed at $X_{1}=0 \AA$, while the other two Gaussians, the positions of which are denoted by $\pm X_{2}$, are mirror images of each other. The anaesthetic density profiles have always been fitted very accurately this way, as shown in Fig. 5, with the exception of the HAL profile at 1000 bar. In this case, the fitting procedure yielded an unphysical (too broad) central Gaussian, its width parameter being comparable with the thickness of the entire membrane. We have regarded this finding as a sign of the loss of the preference of the HAL molecules for being in the middle of the membrane, and hence here we have only used the two Gaussians located at $\pm X_{2}$ in the fitting procedure. The centre of the outer Gaussians, $X_{2}$, as well as the weights of the individual Gaussians in the fitting function $w_{1}$ (being that of the middle Gaussian) and $w_{2}$ (being that of the two outer Gaussians together) are collected in Table 1.
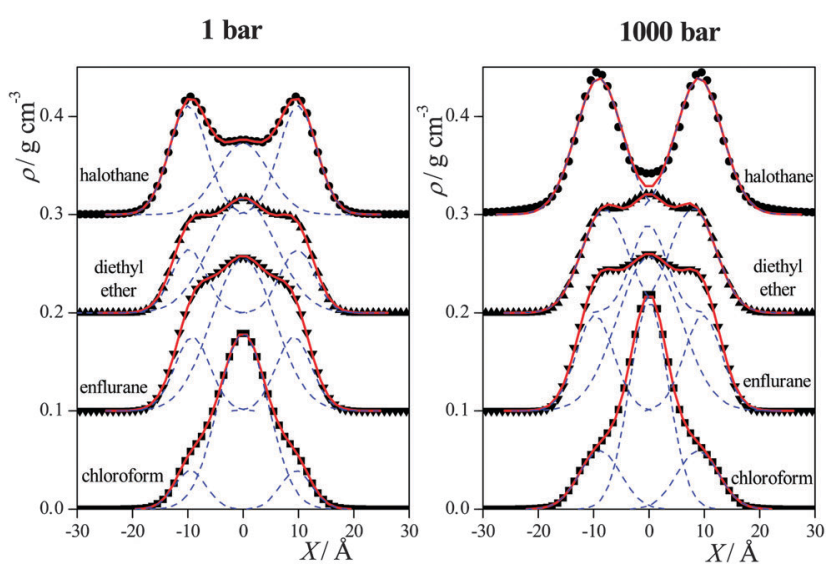

Fig. 5 Mass density profiles of the anaesthetic molecules (full symbols) as obtained at 1 bar (left panel) and 1000 bar (right panel). The profiles of CF, HAL, DE and ENF are shown by squares, circles, up triangles and down triangles, respectively. The sum of three Gaussian functions fitted to these profiles and the individual Gaussians contributing to the total fitting function (see the text) are also shown (red solid lines and blue dashed lines, respectively). The profiles are averaged over the two leaflets of the membrane. Error bars are below $0.01 \mathrm{~g} \mathrm{~cm}^{-3}$. The profiles of ENF, DE, and $\mathrm{HAL}$ are shifted up by $0.1,0.2$ and $0.3 \mathrm{~g} \mathrm{~cm}^{-3}$ for clarity.
Table 1 Peak position and relative weights of the individual Gaussian functions, the sum of which is used to fit the anaesthetic number density profiles (the position of the middle peak is always at $X_{1}=0 \AA$ )

\begin{tabular}{|c|c|c|c|c|c|c|}
\hline \multirow[b]{2}{*}{ Membrane } & \multicolumn{2}{|l|}{$X_{2} / \AA ̊ ̊$} & \multicolumn{2}{|l|}{$w_{1}$} & \multicolumn{2}{|l|}{$\underline{w_{2}}$} \\
\hline & 1 bar & $\begin{array}{l}1000 \\
\text { bar }\end{array}$ & $\begin{array}{l}1 \text { bar } \\
(\%)\end{array}$ & $\begin{array}{l}1000 \\
\text { bar (\%) }\end{array}$ & $\begin{array}{l}1 \text { bar } \\
(\%)\end{array}$ & $\begin{array}{l}1000 \\
\text { bar (\%) }\end{array}$ \\
\hline $\mathrm{DPPC}+\mathrm{CF}$ & 9.6 & 8.8 & 84 & 62 & 16 & 38 \\
\hline DPPC + HAL & 9.9 & 9.5 & 30 & 0 & 70 & 100 \\
\hline DPPC + DE & 9.8 & 8.0 & 63 & 25 & 37 & 75 \\
\hline DPPC + ENF & 9.2 & 9.7 & 65 & 58 & 35 & 42 \\
\hline
\end{tabular}

As is seen, the increase of the pressure has no systematic effect on the location of the outer peaks, however, it clearly leads to the increase of the weight of these outer Gaussians, and to the decrease (and, in the case of HAL, even to the disappearance) of the Gaussian in the middle of the membrane. The weakening of the preference of the anaesthetic molecules for being in the middle of the membrane with increasing pressure is in accordance with the fact that at higher pressures the density in the middle of the membrane is increased, making this position less favourable for the anaesthetic molecules.

\subsection{Average area and volume per lipid}

To test the validity of the critical volume hypothesis, ${ }^{7}$ namely that anaesthesia occurs when the (molar) volume of the cell membrane exceeds a critical value, we have calculated the average surface area of the membrane per DPPC molecule in all the systems simulated, and estimated the volume of the membrane using the peak-to-peak distance of the membrane mass density profile as an estimate of the membrane thickness. The results are summarised in Table 2. The molecular surface area value of $64.3 \AA^{2}$ obtained for the neat DPPC membrane at 1 bar is in a reasonable agreement with the experimental values of $62 \pm 2 \AA^{2},{ }^{58}$ and $62.9 \pm$ $1.3 \AA^{2}, 42$ both measured by Nagle et al.

As is seen, at 1 bar both the average molecular surface area and volume of the membrane have increased noticeably (i.e., by $5-10 \%)$ in the presence of anaesthetic molecules. Further, these values are rather insensitive to the particular choice of the anaesthetic molecule, once the mass density of the anaesthetics is fixed, as in our simulations. Thus, the values obtained with the four anaesthetic molecules considered always agree with each other within $2 \%$ in the case of the average area, and $3 \%$ in the case of the average volume per lipid. Furthermore, the increase of the pressure leads to a substantial, 5-15\% decrease

Table 2 Average molecular surface area and volume of the membranes simulated

\begin{tabular}{lllllr}
\hline & \multicolumn{2}{l}{$\begin{array}{l}\text { Surface area per } \\
\text { DPPC }\left(\AA^{2}\right)\end{array}$} & & \multicolumn{2}{l}{$\begin{array}{l}\text { Membrane volume } \\
\text { per DPPC }\left(\AA^{3}\right)\end{array}$} \\
\cline { 2 - 3 } Membrane & 1 bar & 1000 bar & & 1 bar & 1000 bar \\
\hline Pure DPPC & $64.3 \pm 2.0$ & $58.2 \pm 1.8$ & & $1093 \pm 45$ & $989 \pm 41$ \\
DPPC + CF & $67.1 \pm 2.1$ & $58.4 \pm 1.8$ & & $1191 \pm 49$ & $1051 \pm 43$ \\
DPPC + HAL & $67.0 \pm 2.1$ & $60.8 \pm 1.9$ & & $1156 \pm 48$ & $942 \pm 40$ \\
DPPC + DE & $68.2 \pm 2.2$ & $64.2 \pm 2.0$ & & $1194 \pm 51$ & $1123 \pm 46$ \\
DPPC + ENF & $67.1 \pm 2.1$ & $59.2 \pm 1.9$ & & $1174 \pm 49$ & $1095 \pm 46$
\end{tabular}


of both quantities in every case. Our results are thus in a clear agreement with the critical volume hypothesis of Mullins (although, obviously, they are not enough to prove it). Thus, in systems in which anaesthesia is supposed to occur, the average surface area and volume of the membrane per DPPC molecule are above a certain critical value, being somewhere around $65.5 \AA^{2}$ and $1140 \AA^{3}$, respectively, whereas in those systems in which anaesthesia is not supposed to occur they are below these critical values. In other words, both the average surface area and volume of the membrane per lipid molecule behave in the same way (i.e., increase) upon addition of anaesthetic molecules, irrespective of their chemical form, and behave in the opposite way (i.e., decrease) upon increasing the pressure. Hence, this behaviour can well be related to the molecular mechanism of anaesthesia.

It should be noted, however, that although our results are in accordance with the critical volume hypothesis in general, the details of this behaviour have turned out to be completely different from our simulations than what was previously assumed. Namely, Mullins expected the anaesthetic molecules to increase the thickness of the membrane, without altering its lateral density. ${ }^{7}$ Our results, on the other hand, have revealed an opposite behaviour: whilst the peak-to-peak distance (i.e., thickness) of the membrane is found to be insensitive both to the presence/absence of the anaesthetic molecules and to the pressure (see the discussion in the previous subsection), the lateral density of the membrane (i.e., its average area per lipid) behaves in exactly the same way as the molar volume. In this sense, contrary to the original hypothesis of Mullins, based on a critical membrane thickness, the critical volume behaviour observed here is based on a critical surface density phenomenon.

\subsection{Self-association of anaesthetics}

Another possible explanation of the molecular mechanism of anaesthesia and its pressure reversal is based on the claim that at high pressure values the anaesthetic molecules form selfassociates, and thus lose their ability to act as anaesthetics. ${ }^{27,31}$ To check the validity of this assumption, we have calculated the size distribution of the aggregates formed by the anaesthetic molecules at different pressures. Two anaesthetic molecules are considered here as being "bound" to each other if the distance of their centre-of-mass is below the first maximum position of the corresponding radial distribution function, i.e., the two molecules are in the first coordination shell of each other. According to the respective radial distribution functions these cut-off distances have been chosen as $7.9 \AA$, 9.0 ̊, $6.9 \AA$, and $6.5 \AA$ for CF, HAL, DE and ENF, respectively. ${ }^{32}$ Two anaesthetic molecules are then regarded as belonging to the same selfassociate if they are linked together via bound molecule pairs.

The obtained self-associate cluster size distributions are shown in Fig. 6 for all the four anaesthetic molecules. For completeness, this figure includes even the results we obtained at 100 bar as well as with HAL at lower concentration. As is seen, at least some of the anaesthetic molecules do not show any particular preference for self-association. Thus, for instance, more than $80 \%$ of the ENF molecules are isolated from each

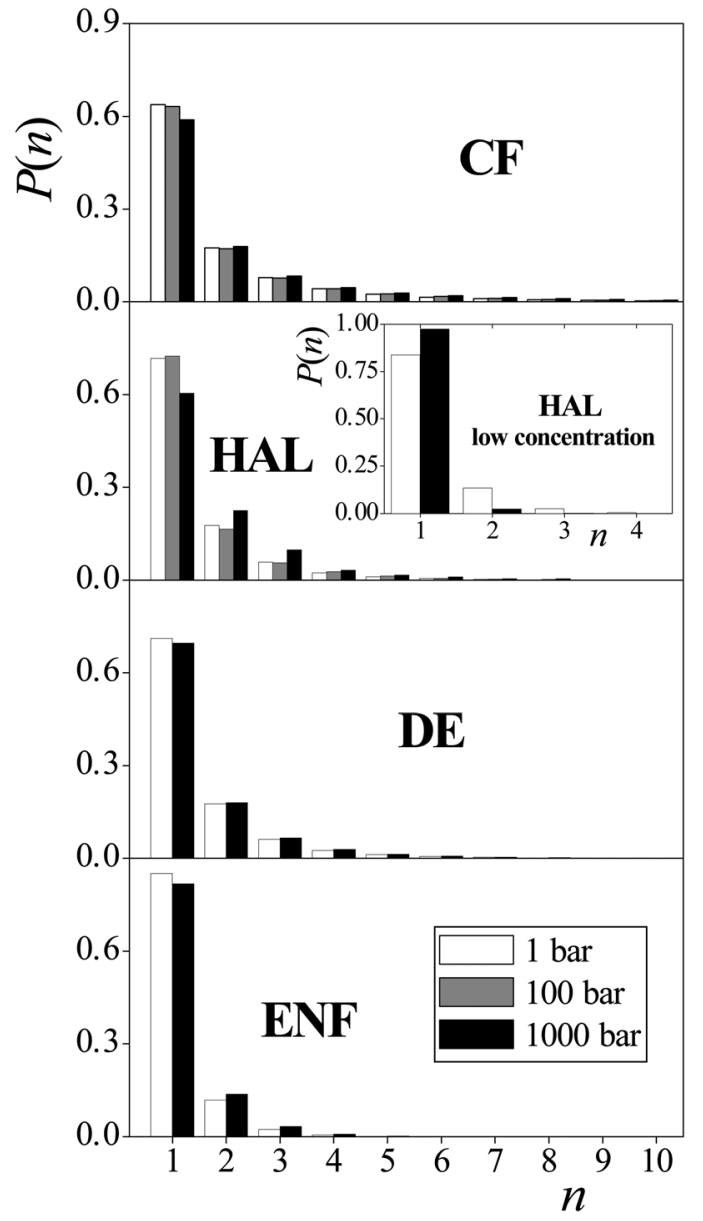

Fig. 6 Size distribution of the self-associates formed by the anaesthetic molecules: CF (top panel), HAL (second panel), DE (third panel) and ENF (bottom panel) in the membrane at 1 bar (white columns), 100 bar (grey columns) and 1000 bar (black columns). The inset shows the results obtained using half of the HAL concentration. Error bars are below 0.001 units.

other at both pressures. More importantly, the tendency of the different anaesthetic molecules for self-association clearly turns out to be pressure independent, as the associate size distributions obtained at different pressures are practically identical to each other for all the four anaesthetic molecules considered. This finding clearly reveals that self-association of the anaesthetic molecules can be ruled out as a possible molecular reason of anaesthesia, as it cannot account for its pressure reversal.

\subsection{Lipid orientation}

3.4.1. Orientation of lipid tails and heads. Another frequent claim concerning the molecular background of anaesthesia is that in the presence of anaesthetic molecules the lipid tails are, on average, in more extended conformations, in other words, anaesthetics have an ordering effect on the lipid tails. ${ }^{13,19,20}$ This idea is behind, among others, the aforementioned critical volume hypothesis of Mullins, ${ }^{7}$ as the increase of the membrane thickness in the presence of anaesthetics is supposed to occur right because of this straightening of the lipid tails. To test this idea, we have calculated the cosine distribution of the angle $\gamma$, 
formed by the membrane normal vector, $\boldsymbol{X}$ (pointing, to our convention, towards the middle of the membrane), and the vector describing the overall orientation of the lipid tail. This latter vector is defined as pointing from the first $\mathrm{CH}_{2}$ group of the tail (marked by 1 in Fig. 1) to the chain terminal $\mathrm{CH}_{3}$ group. For completeness, we have also calculated the cosine distribution of the angle $\alpha$, formed by the membrane normal vector, $\boldsymbol{X}$, and the vector pointing from the $\mathrm{P}$ to the $\mathrm{N}$ atom of the DPPC headgroup (since these two atoms are the centres of the negative and positive charges, this vector roughly estimates the dipole moment of the zwitterionic headgroup of DPPC).

The cosine distributions obtained in the systems simulated are shown in Fig. 7. As is seen, the distribution of $\cos \alpha$, describing the headgroup orientation, is rather insensitive both to the presence of anaesthetic molecules and to the pressure. The $\mathbf{P N}$ vector prefers to lie parallel with the membrane, in order to allow the dipole vectors of the neighbouring headgroups taking relative alignments in which their electrostatic attraction is sufficiently large. It should be noted that in real biological membranes the lipid headgroups are in contact with physiological electrolyte solution rather than neat water, as in the case of our simulation, which might well affect the observed orientational preferences. However, what is important from our purpose is that neither the pressure nor the presence of the anaesthetic molecules have a noticeable effect on these distributions.

Unlike in the case of the headgroups, the increase of the pressure indeed has an overall straightening effect on the lipid tails. However, the tail orientation, similar to that of the headgroups, is again found to be completely insensitive to the presence or absence of the anaesthetic molecules in the system. This finding is also in a clear accordance with the previously discussed insensitivity of the peak-to-peak distance of the mass density profiles (i.e., membrane thickness) to the presence of the anaesthetic molecules. Therefore, changes in the orientation of different parts of the lipid molecules cannot be related to the molecular reasons lying behind the phenomenon of anaesthesia, either.

3.4.2. Deuterium order parameters. Although the overall orientation of the lipid tails turned out to be insensitive to the presence or absence of anaesthetic molecules, the local order of the tails might still well depend on them. To investigate this point, we have determined the deuterium order parameter profile of the lipid tails in the systems simulated. The deuterium order parameter, $S_{\mathrm{CD}}$, accessible experimentally by nuclear magnetic resonance (NMR) measurements of selectively deuterated samples, describes the local orientational order of the hydrocarbon tail around its individual $\mathrm{CH}_{2}$ groups. $S_{\mathrm{CD}}$ is defined as ${ }^{59,60}$

$$
S_{\mathrm{CD}}=\frac{2 S_{x x}+S_{y y}}{3},
$$

where the $S_{i j}$ element of the order parameter tensor can be calculated as

$$
S_{i j}=\frac{\left\langle 3 \cos \vartheta_{i} \cos \vartheta_{j}-\delta_{i j}\right\rangle}{2} .
$$

Here $\delta_{i j}$ is the Kronecker delta, whereas $\vartheta_{i}$ and $\vartheta_{j}$ denote the angles formed by the membrane normal axis, $X$, and the $i$ th and $j$ th axis,
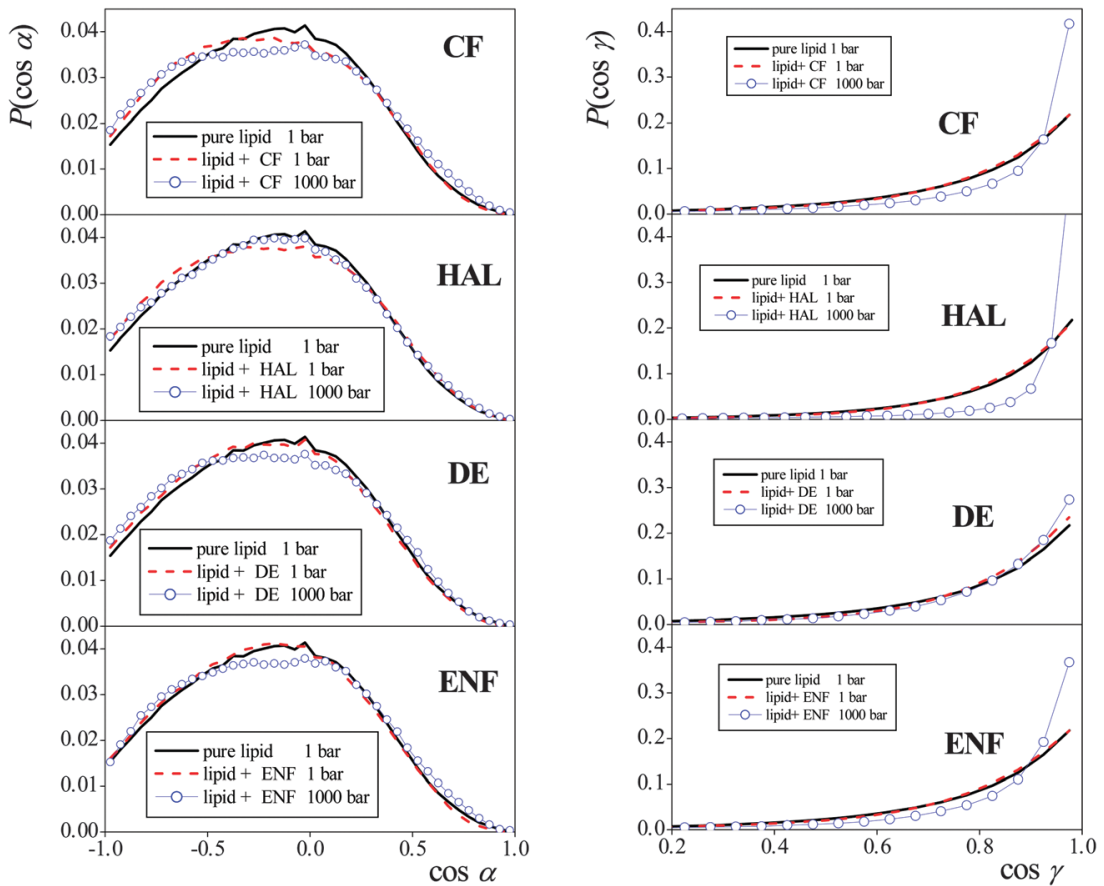

Fig. 7 Cosine distributions of the angles $\alpha$ (left) and $\gamma$ (right) formed by the membrane normal vector, $\boldsymbol{X}$ (pointing towards the middle of the membrane), with the vectors pointing from the headgroup P to $\mathrm{N}$ atom, and from the first to the last tail $\mathrm{C}$ atom, respectively, of the DPPC molecules. Black solid lines: the results in the pure DPPC membrane at 1 bar, red dashed lines: the results in the anaesthetic containing membrane at 1 bar, blue lines with open circles: the results in the anaesthetic containing membrane at 1000 bar. The top, second, third, and fourth panels correspond to membranes containing $C F, H A L, D E$, and ENF, respectively. 
respectively, of the molecule-fixed, local Cartesian frame. Since in our simulations the $\mathrm{CH}_{2}$ and $\mathrm{CH}_{3}$ groups are treated as united atoms, this local frame is defined in the following way. ${ }^{59,60}$ Its origin is the $\mathrm{C}$ atom in question along the hydrocarbon tail, its axis $z$ connects the $\mathrm{C}$ atoms preceding and following it along the chain, axis $y$, being perpendicular to $z$, still lies in the plane defined by these three $\mathrm{C}$ atoms, while axis $x$ is perpendicular to the above two. Clearly, the $S_{\mathrm{CD}}$ parameter can thus be not defined for the first and last $\mathrm{C}$ atoms of the lipid tails.

The order parameter profiles obtained in the systems simulated are shown and compared to each other in Fig. 8. The numbering scheme of the $\mathrm{C}$ atoms used along the tails is shown in Fig. 1. The inset shows a comparison of the $S_{\mathrm{CD}}$ profile obtained here for the neat lipid membrane at 1 bar with that obtained previously at $300 \mathrm{~K},{ }^{32}$ and also with the experimental data of Douliez et al., obtained in the liquid crystalline $\left(\mathrm{L}_{\alpha}\right)$ phase of DPPC, at $338 \mathrm{~K}$ by NMR measurements. ${ }^{61}$ As is seen, the present results agree very well with the experimental data, giving thus some additional confidence of the present results. More importantly, the shape of the simulated profiles obtained at different temperatures are characteristically different from each other in the region of the first few carbon atoms, indicating that the two simulated membranes are in different phases, and hence the present results are indeed obtained in the biologically more relevant liquid crystalline $\left(\mathrm{L}_{\alpha}\right)$ phase.

As is seen from Fig. 8, in accordance with the overall orientation of the lipid tails, pressure has a clear and marked effect on the order parameter profile, as its increase has led to a clear increase of the $S_{\mathrm{CD}}$ parameter along the entire tail in every case. No such clear effect of the presence of the anaesthetic molecules is, however, seen at the $S_{\mathrm{CD}}$ profiles. Nevertheless, at $1 \mathrm{bar}$, the presence of all anaesthetic molecules considered has

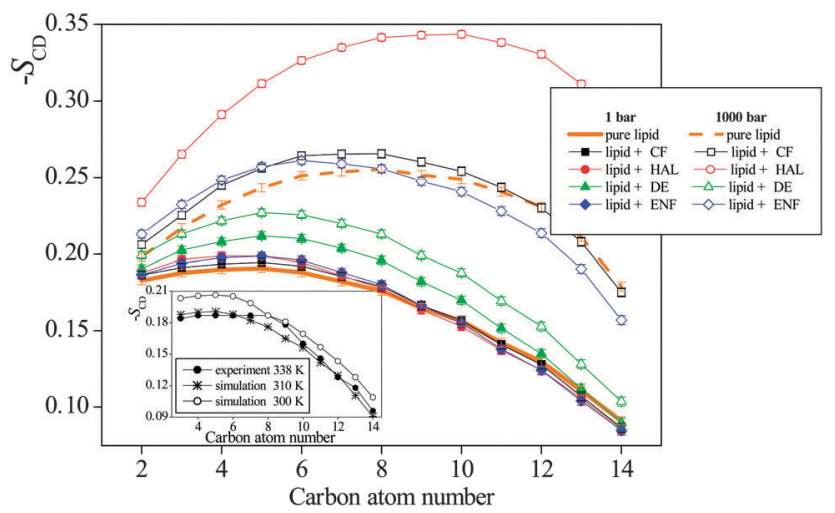

Fig. 8 Deuterium order parameter profiles of the $\mathrm{CH}_{2}$ groups along the DPPC tails (averaged over the two tails of the DPPC molecule), as obtained in the neat DPPC membrane (thick orange lines) at 1 bar (solid line) and 1000 bar (dashed line), and in the membranes containing CF (black squares), HAL (red circles), DE (green triangles), and ENF (blue diamonds) at 1 bar (full symbols) and 1000 bar (open symbols). The inset shows a comparison of the simulated $S_{C D}$ profile of the neat DPPC membrane at atmospheric pressure as obtained at $310 \mathrm{~K}$ (asterisks) and $300 \mathrm{~K}$ (open circles) ${ }^{32}$ with the experimental data of Douliez et al., obtained at $338 \mathrm{~K}$ (full circles). ${ }^{61}$ The lines connecting the symbols are just guide to the eye. Error bars are only shown when larger than the symbols. turned out to increase the orientational order of the tails up to about the eighth $\mathrm{C}$ atom, although at 1000 bar the addition of DE to the membrane has led to the decrease of $S_{\mathrm{CD}}$ all along the entire tail. The ordering effect of the anaesthetic molecules in this part of the tail is clearly related to the fact that these tail $\mathrm{C}$ atoms are located in the $X$ range corresponding to one of the two preferred locations of the anaesthetics. On the other hand, in the membrane interior, i.e., beyond the eighth $\mathrm{C}$ atom, no clear effect of the anaesthetics is observed on the $S_{\mathrm{CD}}$ order parameter profile.

Although all anaesthetic molecules considered here have the same, though weak effect on the local orientational order of the lipid tails, at least in the region of their first few $\mathrm{C}$ atoms, this effect cannot be related again to the molecular mechanism of anaesthesia, as the increase of the pressure leads to much more pronounced changes to the same direction (i.e., causes a further increase of the local tail order), and hence these changes are incompatible with the phenomenon of pressure reversal of anaesthesia.

Finally, to check whether the presence of anaesthetic molecules might lead to the systematic decrease of the local orientational order of at least some of the lipid tails, we have calculated the $S_{\mathrm{CD}}$ order parameter profiles in the anaesthetic containing systems at 1 bar separately for the lipids that are close to an anaesthetic molecule and that are far from anaesthetic molecules. In this respect, a DPPC and an anaesthetic molecule have been considered to be close to each other if the lateral distance (i.e., distance within the $Y Z$ plane) of their centre-of-mass is less than $8.3 \AA$, the first minimum position of the corresponding radial distribution function. ${ }^{32}$ The order parameter profiles obtained in this way are shown and compared to that of the neat DPPC membrane in Fig. 9. Although the local orientational order of the tails that are close to an anaesthetic molecule is, in general, somewhat lower than that of those being far from anaesthetics, in some cases (e.g., in the presence of $\mathrm{DE}$ ) the order of both types of tails is clearly larger than in the absence of anaesthetics. Therefore, changes even in the local orientational order of the lipid tails can be excluded from the possible molecular reasons that might be behind the phenomenon of anaesthesia.

\section{Summary and conclusions}

In this paper we performed a systematic study of the effect of general anaesthetic molecules as well as of high pressure on the properties of a DPPC membrane in the biologically most relevant liquid crystalline $\left(\mathrm{L}_{\alpha}\right)$ phase. Our approach was that only such membrane properties can possibly be related to the molecular mechanism of anaesthesia that are (i) changed in the same way by the presence of all general anaesthetic molecules, and (ii) are changed in the opposite way by the increase of the pressure, to be consistent also with the phenomenon of pressure reversal. Neither the density profiles of the entire membrane as well as of selected lipid groups or anaesthetic molecules, nor the orientation of the lipid tails and headgroups, nor the deuterium order parameter profile of the lipid 


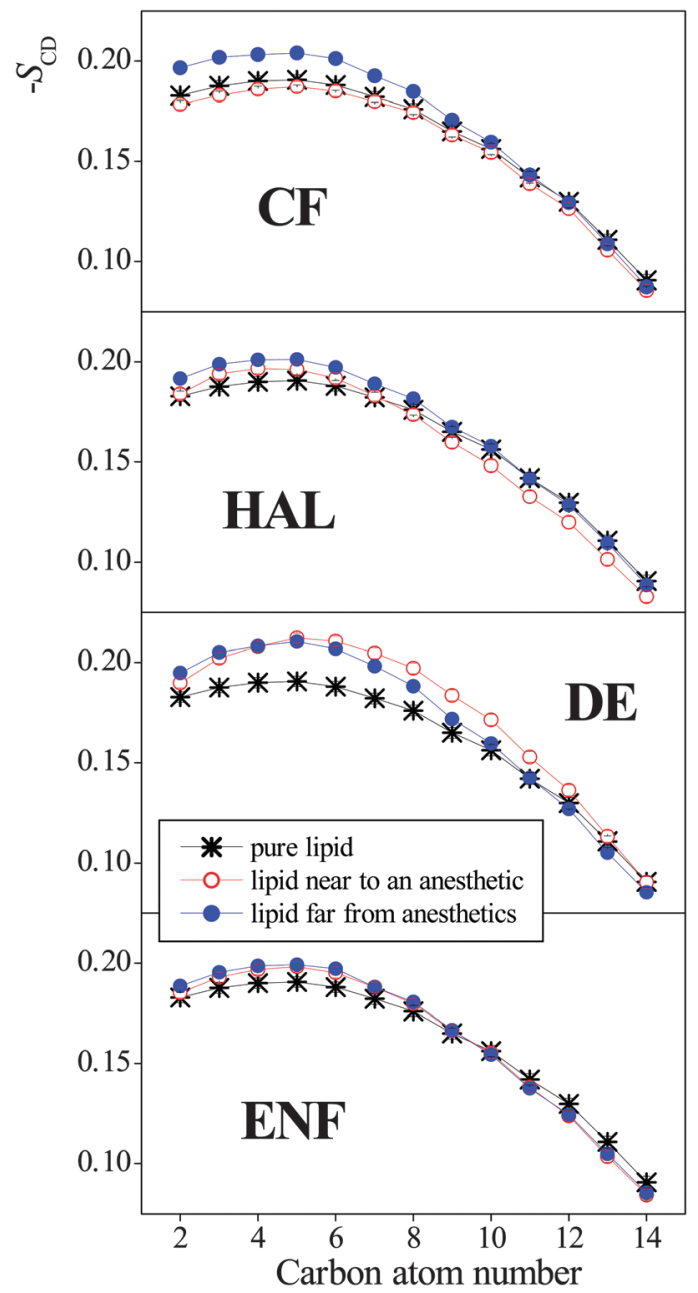

Fig. 9 Deuterium order parameter profiles of the $\mathrm{CH}_{2}$ groups along the DPPC tails (averaged over the two tails of the DPPC molecule), as obtained at 1 bar in the neat DPPC membrane (black asterisks), and in the anaesthetic containing membrane for the lipid molecules that are close to an anaesthetic molecule (red open circles) and that are far from the anaesthetic molecules (blue full circles). For the definition of lipids being close to or far from anaesthetics, see the text. The top, second, third, and fourth panels correspond to membranes containing CF, HAL, DE, and ENF, respectively. The lines connecting the symbols are just guide to the eye. Error bars are only shown when larger than the symbols.

tails, characterising the local orientational order turned out to be such a property, and thus their changes can be ruled out as possible molecular reasons behind the phenomenon of anaesthesia. Furthermore, the self-association of the anaesthetic molecules turned out to be completely pressure independent, and hence it cannot be related to anaesthesia, either. In fact, we only found one membrane property, namely the average membrane volume per lipid molecule that fulfils both of the above conditions. When the molecular volume of the membrane was decomposed to the surface area per lipid and membrane thickness, it turned out that while the membrane surface area also follows our above two rules, i.e., increases in the presence of anaesthetics and decreases at high pressure, the membrane thickness does not show a clear dependence on the presence of anaesthetic molecules and pressure.
Our finding is in accordance with the more than half a century old critical volume hypothesis of Mullins, ${ }^{7}$ however, the origin of this critical volume behaviour turned out to be different than what was originally hypothesized. Namely, the membrane volume per lipid exceeds a critical value in all cases when anaesthesia is supposed to occur, and remains below this critical value otherwise because of the similar behaviour of the membrane surface area, and not the membrane thickness, as it was assumed by Mullins. ${ }^{7}$ All these results indicate that the anaesthetic-induced lateral expansion and pressure-induced lateral contraction of the membrane can well be behind the molecular mechanism of anaesthesia and its pressure reversal (although, obviously, the present study cannot prove a causal relation between them).

Another important finding of the present work is that general anaesthetic molecules have a dual preference for locations along the membrane normal axis, although the relative strengths of these two preferences can change strongly from one anaesthetic to the other. Nevertheless, all the four anaesthetic molecules prefer to stay right in the middle of the membrane, and also about $10 \AA$ away from it, close to the polar headgroups, yet in the hydrocarbon region. The former position is stabilised by steric effects (low density in the middle of the membrane), while the latter position is likely to be stabilised by the interaction between the headgroup region and the weakly polar anaesthetic molecules. In the case of halothane, this position is further stabilised by the formation of weak $\mathrm{C}-\mathrm{H}$...O type hydrogen bonds between the halothane $\mathrm{CH}$ groups and ester $\mathrm{O}$ atoms of the lipids. The increase of the pressure led to the relative weakening of the preference for the former, and strengthening of that for the latter position, possibly by increasing the density in the middle of the membrane. Therefore, the pressure-induced changes of this dual preference might also be related to the pressure reversal of anaesthesia; if this is indeed the case then the phenomenon of anaesthesia itself must be caused by those anaesthetic molecules that are located in the middle of the membrane.

\section{Acknowledgements}

This project is supported by the Hungarian OTKA Foundation under project No. 104234, by the Action Austria Hungary Foundation under project No. 90öu18, and by CNRS in the framework of an international program for scientific cooperation (PICS).

\section{References}

1 H. Meyer, Naunyn-Schmiedebergs Archiv für Experimentelle Pathologie und Pharmakologie, 1899, vol. 42, p. 109.

2 E. Overton, Studien über die Narkose zugleich ein Beitrag zur allgemeinen Pharmakologie, Gustav Fischer Verlag, Jena, 1901.

3 F. H. Johnson and E. A. Flagler, Science, 1950, 112, 91.

4 M. J. Lever, K. W. Miller, W. D. M. Paton and E. B. Smith, Nature, 1971, 231, 368.

5 M. J. Halsey and B. Wardley-Smith, Nature, 1975, 257, 811. 
6 J. R. Trudell, D. G. Payan, J. H. Chin and E. N. Cohen, Proc. Natl. Acad. Sci. U. S. A., 1975, 72, 210.

7 L. J. Mullins, Chem. Rev., 1954, 54, 289.

8 S. M. Johnson and K. W. Miller, Nature, 1970, 228, 75.

9 M. K. Jain, N. Y. Wu and L. V. Wray, Nature, 1975, 255, 494.

10 K. W. Miller and K. Pang, Nature, 1976, 263, 253.

11 J. M. Boggs, T. Yoong and J. C. Hsia, Mol. Pharmacol., 1976, $12,127$.

12 D. A. Haydon, B. M. Hendry, S. R. Levinson and J. Requena, Nature, 1977, 268, 356.

13 R. G. Ashcroft, H. G. L. Coster and J. R. Smith, Nature, 1977, 269, 819.

14 C. Y. Kao, Pharmacol. Rev., 1966, 18, 987.

15 A. G. Lee, Nature, 1976, 262, 545.

16 N. P. Franks and W. R. Lieb, Nature, 1978, 274, 339.

17 N. P. Franks and W. R. Lieb, Nature, 1994, 367, 607.

18 D. C. Mitchell, J. T. R. Lawrence and B. J. Litman, J. Biol. Chem., 1996, 32, 19033.

19 R. G. Ashcroft, H. G. L. Coster and J. R. Smith, Biochim. Biophys. Acta, 1977, 469, 13.

20 D. A. Haydon, B. M. Hendry, S. R. Levinson and J. Requena, Nature, 1977, 268, 356.

21 B. J. Forrest and D. K. Rodham, Biochim. Biophys. Acta, 1985, 814, 281.

22 R. S. Cantor, Biochemistry, 1997, 36, 2339.

23 N. P. Franks and W. R. Lieb, J. Mol. Biol., 1979, 133, 469.

24 G. L. Turner and E. Oldfield, Nature, 1979, 277, 669.

25 J. R. Trudell, W. L. Hubbell and E. N. Cohen, Biochim. Biophys. Acta, 1973, 291, 321.

26 P. Huang, E. Bertaccini and G. H. Loew, J. Biomol. Struct. Dyn., 1995, 12, 725.

27 P. L. Chau, P. N. M. Hoang, S. Picaud and P. Jedlovszky, J. Mol. Liq., 2009, 147, 128.

28 K. J. Oh and M. L. Klein, Bull. Korean Chem. Soc., 2009, 30, 2087.

29 J. J. López Cascales, J. G. Hernández Cifre and J. García de la Torre, J. Phys. Chem. B, 1998, 102, 625.

30 L. Koubi, M. Tarek, M. L. Klein and D. Scharf, Biophys. J., 2000, 78, 800.

31 P. L. Chau, P. N. M. Hoang, S. Picaud and P. Jedlovszky, Chem. Phys. Lett., 2007, 438, 294.

32 M. Darvas, P. N. M. Hoang, S. Picaud, M. Sega and P. Jedlovszky, Phys. Chem. Chem. Phys., 2012, 14, 12956.

33 B. Griepernau and R. A. Böckmann, Biophys. J., 2008, 95, 5766.

34 P. Jedlovszky and M. Mezei, J. Am. Chem. Soc., 2000, $122,5125$.

35 P. Jedlovszky and M. Mezei, J. Phys. Chem. B, 2003, 107, 5322.

36 K. Tu, M. Tarek, M. L. Klein and D. Scharf, Biophys. J., 1998, 75, 2123.
37 M. Pickholz, L. Saiz and M. L. Klein, Biophys. J., 2005, 88, 1524.

38 L. J. Martin, R. Chao and B. Corry, Biophys. Chem., 2014, 185, 98.

39 N. Hauet, F. Artzner, F. Boucher, C. Grabielle-Madelmont, I. Cloutier, G. Keller, P. Lesieur, D. Durand and M. Paternostre, Biophys. J., 2003, 84, 3123.

40 R. D. Porasso, W. F. Drew Bennett, S. D. Oliveira-Costa and J. J. López Cascales, J. Phys. Chem. B, 2009, 113, 9988.

41 C. Anézo, A. H. de Vries, H. D. Höltje, D. P. Tieleman and S. J. Marrink, J. Phys. Chem. B, 2003, 107, 9424.

42 J. F. Nagle, R. Zhang, S. Tristram-Nagle, W. Sun, H. I. Petrache and R. M. Suter, Biophys. J., 1996, 70, 1419.

43 O. Berger, O. Edholm and F. Jähnig, Biophys. J., 1997, 72, 2002.

44 S. Leekumjorn and A. K. Sum, Biochim. Biophys. Acta, 2007, 1768, 354.

45 H. J. C. Berendsen, J. P. M. Postma, W. F. van Gunsteren and J. Hermans, in Intermolecular Forces, ed. B. Pullman, Reidel, Dordrecht, 1981, p. 331.

46 D. Scharf and K. Laasonen, Chem. Phys. Lett., 1996, 258, 276. 47 W. F. van Gunsteren, S. R. Billeter, A. A. Eising, P. H. Hünenberger, P. Krüger, A. E. Mark, W. R. P. Scott and I. G. Tironi, Biomolecular Simulation: The GROMOS96 Manual and User Guide, vdf Hochschulverlag A.G. an der ETH Zürich and BIOMOS b.v., Zürich, Groningen, 1996.

48 L. D. Schuler, X. Daura and W. F. van Gunsteren, J. Comput. Chem., 2001, 22, 1205.

49 A. W. Schuettelkopf and D. M. F. van Aalten, Acta Crystallogr., Sect. D: Biol. Crystallogr., 2004, 60, 1355.

50 C. Oostenbrink, A. Villa, A. E. Mark and W. F. van Gunsteren, J. Comput. Chem., 2004, 25, 1656.

51 B. Hess, H. Bekker, H. J. C. Berendsen and J. G. E. M. Fraaije, J. Comput. Chem., 1997, 18, 1463.

52 S. Miyamoto and P. A. Kollman, J. Comput. Chem., 1992, 13, 952.

53 U. Essman, L. Perera, M. L. Berkowitz, T. Darden, H. Lee and L. G. Pedersen, J. Chem. Phys., 1995, 103, 8577.

54 B. Hess, C. Kutzner, D. van der Spoel and E. Lindahl, J. Chem. Theory Comput., 2008, 4, 435.

55 S. Nosé, Mol. Phys., 1984, 52, 255.

56 W. G. Hoover, Phys. Rev. A: At., Mol., Opt. Phys., 1985, 31, 1695.

57 M. Parinello and A. Rahman, J. Appl. Phys., 1981, 52, 7182. 58 J. F. Nagle, Biophys. J., 1993, 64, 1476.

59 J. J. López-Cascales, J. García de la Torre, S. J. Marrink and H. J. C. Berendsen, J. Chem. Phys., 1996, 104, 2713.

60 M. Mezei and P. Jedlovszky, in Methods in Molecular Biology Methods in Membrane Lipids, ed. A. M. Dopico, Humana Press, Totowa, NJ, 2007, vol. 400, pp. 127-144.

61 J. P. Douliez, A. Léonard and E. J. Dufourc, Biophys. J., 1995, 68, 1727. 